\title{
Viabilidad económica de la producción de espárrago en la Región del Maule, Chile
}

\author{
Germán L Andrade ${ }^{1}$; Tristán M Ibáñez ${ }^{2}$ \\ ${ }^{1}$ Universidad de Talca - Facultad de Ciencias Empresariales, P.O. 721, Talca, Chile; ${ }^{2}$ Universidad Austral de Chile, P.O. 542, Linares, \\ Chile. E-mail: globos@utalca.cl;curimaqui@tie.cl
}

\section{RESUMEN}

En esta investigación se estimaron indicadores de viabilidad y de eficiencia económica de la producción de espárrago (Asparagus officinalis L.) en Chile. Los coeficientes técnicos fueron tomados de los registros históricos de un predio localizado en la zona de Linares, Región del Maule, Chile. Los indicadores de viabilidad calculados fueron el valor actual neto (VAN), tasa interna de retorno (TIR), índice de valor actual neto (IVAN), razón beneficio-costo (RBC), y período de recuperación del capital (PRC). Los indicadores de eficiencia económica fueron el costo medio total (CMET), margen unitario ponderado (MUP) y la rentabilidad sobre capital (RSC). Como unidad de análisis se consideró una hectárea. Para productores con tierra, los resultados obtenidos en la situación estándar fueron: Ch\$1,52 millones (VAN), 12,1\% (TIR), 0,3 (IVAN), 1,1 (RBC) y 10 años (PRC). Para la etapa de producción se proyectó un CMET mínimo de Ch $\$ 317,8 \mathrm{~kg}^{-1}$, un MUP máximo de $\mathrm{Ch} \$ 407 \mathrm{~kg}^{-1}$ y una RSC de 20,3\%. La principal conclusión es que el cultivo de espárrago en la región del Maule, en Chile, es viable para los productores con tierra.

Palabras-clave: Asparagus officinalis L., valor actual neto, tasa interna de retorno, razón beneficio-costo.

\begin{abstract}
Economic viability of asparagus production in the Maule Region, Chile

Viability and economic efficiency indicators for asparagus (Asparagus officinalis L.) production in Chile were evaluated. Technical parameters were obtained from an historical farm located in Linares area, Maule Region, Chile. Viability was assessed by means of net present value (VAN), internal rate of return (TIR), net present value index (IVAN), cost-benefit ratio (RBC), and investment recovery period (PRC). Economic efficiency was assessed through production average cost (CMET), weighted unit margin (MUP), and return on equity (RSC). The analysis was based upon one hectare. Results for producers who own their land were US\$2,529 ha-1 (VAN), $12.14 \%$ (TIR), 0.25 (IVAN), and 1.1 (RBC). The minimum CMET was estimated at US\$0.53 $\mathrm{kg}^{-1}$ the maximum MUP at US\$0.68 kg-1 , and RSC until $20.3 \%$. The main conclusion is that asparagus production in the Maule region, in Chile, is viable for producers who own their land.
\end{abstract}

Keywords: Asparagus officinalis L., net present value, internal rate return, cost-benefit ratio.

\section{(Recebido para publicação em 17 de fevereiro de 2005; aceito em 3 de maio de 2006)}

A nivel mundial los principales productores de espárrago (Asparagus officinalis L.) son China, Perú, EE.UU. y México. En el año 2003, la producción de China alcanzó a 5,5 millones de $\mathrm{t}$ ( $87 \%$ de la producción total del mundo), seguido de Perú (185.000 t, 3\%), los EE.UU. (122.020 t, 2\%) y México $(67.247$ t, 1\%); mientras la producción de Chile alcanzó a 19.500 t, alrededor del $0,3 \%$ de la producción mundial. Además, durante el período 1999 a 2003 se observó un incremento en la producción mundial del $60 \%$, llegando a 6,3 millones de t (FAOSTAT, 2004). China produce principalmente espárrago blanco, el cual se comercializa fundamentalmente en el mercado doméstico. El espárrago verde es producido en cantidades limitadas. En Perú alrededor del $40 \%$ de la producción corresponde a espárrago verde, el cual se comercializa en fresco, mientras que el restante $60 \%$ corresponde a espárrago blanco, el cual es procesado y enviado a los mercados de la Unión Europea; los EE.UU. y México producen principalmente cultivares verdes (USDA, 2004). De acuerdo con cifras de Global Trade Information (GTI, 2004), durante el año 2003 los principales exportadores de espárrago fresco fueron Perú (66.844 t), México (47.657 t) y EE.UU. (23.675 t); mientras los principales importadores fueron EE.UU. (96.455 t), Unión Europea -15 (21.905 t) y Japón (17.850 t).

En el mercado internacional se comercializa principalmente el cultivar Mary Washington, a partir de la cual se han derivado varios híbridos, denominados UC, desarrollados por la Universidad de California. Entre ellos destaca el híbrido clonal UC-157F1, el cual ocupa prácticamente el $90 \%$ de la superficie cultivada en Chile. En menor medida se utilizan los cultivares Atlas y Apollo (González, 1999).

Durante el año 2003, la superficie cultivada con espárrago en Chile fue de
4.300 ha (FAOSTAT, 2004), las exportaciones alcanzaron a $6.850 \mathrm{t}$ y generaron retornos por US\$13,2 millones (ODEPA, 2004). Los precios promedio ponderado FOB fluctuaron entre US\$ 1,29 y $1,69 \mathrm{~kg}^{-1}$ durante el período 2000 a 2003. En el mercado interno los precios promedio a nivel mayorista fluctuaron entre $\mathrm{Ch} \$ 490,7$ y $579,2 \mathrm{~kg}^{-1}$ (equivalente a un rango entre US $\$ 0,82$ y $0,97 \mathrm{~kg}^{-1}$ ) durante el mismo período.

A nivel internacional, la literatura reporta diversas investigaciones respecto a la influencia de diferentes densidades de plantación sobre los rendimientos. Algunas densidades consideradas han sido entre $41.700 \mathrm{y}$ 50.000 plantas ha $^{-1}$ (Ferrari et al., 1990), 30.000 y 45.000 plantas ha $^{-1}$ (Kaufmann \& Orth, 1990), entre 33.333 y 66.667 plantas ha-1 (Loughton et al., 1996), y entre 8.500 y 66.000 plantas $\mathrm{ha}^{-1}$ (Bussell et al., 1997). En el caso de Chile, para el mismo cultivar de 
espárrago considerado en este trabajo, González \& Del Pozo (2003), estudiaron el efecto de tres profundidades y dos densidades de plantación sobre el rendimiento y calidad del espárrago verde en la zona climática mediterránea sub-húmeda. En el caso de la plantación más superficial $(10 \mathrm{~cm})$, los autores observaron un mayor rendimiento comercial sólo durante los primeros cuatro años después de la plantación y un menor calibre después de cinco años de cosecha; además, la mayor densidad de plantación (33.333 plantas/ha) presentó un rendimiento más alto en todas las temporadas. En todos los casos, el trabajo de González \& Del Pozo (2003), reportó un rendimiento exportable superior al $80 \%$. En otros estudios de densidad de plantación realizados en Chile con el cultivar UC$157 \mathrm{~F} 1$ no fueron encontradas diferencias en el rendimiento después de la tercera temporada de cosecha (Pignon et al., 1994; González \& Del Pozo, 1999).

La mayor parte de las plantaciones de espárrago en Chile se encuentran bajo un régimen de clima mediterráneo, es decir, zonas donde el invierno es relativamente frío y lluvioso, la primavera es cálida y húmeda, y el verano es caluroso y seco (Del Pozo, 1999). Según Ortega (1999), el suelo ideal para el establecimiento de la esparraguera es de textura franco arenosa, de buen drenaje, buena estructura, con un contenido mínimo de $2 \%$ de materia orgánica, y una profundidad apropiada (mínimo $1 \mathrm{~m}$ ) para el crecimiento de las raíces del cultivo. El pH ideal para el cultivo es entre 6,5 y 7,5. Las labores necesarias para realizar la plantación de una esparraguera se deben iniciar en agosto con la roturación del suelo y finalizan en junio del año siguiente con el retiro de cañas secas; la mejor época de plantación es en septiembre (Velasco \& Avilés, 1999). En Chile, el período productivo de una esparraguera debiera superar los 10 años en una plantación comercial, si ha sido bien manejada (Del Pozo, 1999). En general, la cosecha del espárrago se realiza entre septiembre y principios de diciembre, aunque es habitual extenderse en la cosecha de esparragueras más antiguas hasta fines de diciembre (Del Pozo, 1999). En general, las enfermedades radiculares son las principales causales de pérdida de vigor y muerte de plantas de espárrago, mientras que las enfermedades foliares causan disminución de calidad (France, 1999).

El objetivo de esta investigación fue analizar el desempeño económico de la producción de espárrago verde, cultivar UC-157F1, así como estimar indicadores de viabilidad y eficiencia económica en un cultivo en la provincia de Linares, Región del Maule, Chile.

\section{MATERIALES Y MÉTODOS}

Los coeficientes técnicoeconómicos utilizados para la valorización de las inversiones y costos fueron tomados de los registros históricos (1995/1996 a 2003/2004) de un predio de 40 ha localizado en la provincia de Linares $\left(35^{\circ} 51^{\prime}\right.$ Lat. S. y 71³5' Long. O), Región del Maule, Chile. El terreno agrícola tomado como base para las estimaciones cumple con las aptitudes edafoclimáticas para la plantación de espárragos. Como unidad de análisis se consideró 1 ha y una densidad de plantación de 40.000 plantas $\mathrm{ha}^{-1}$, en hileras dobles de $0,2 \mathrm{x}$ $0,2 \mathrm{~m}$ sobre hilera, y distanciadas a 2,5 $\mathrm{m}$ entre hileras. Para efectos del proyecto se definió un horizonte de evaluación de 10 años, incluyendo los dos primeros años (etapas de establecimiento y formación del cultivo).

Para estimar la viabilidad económica se calcularon los siguientes indicadores: valor actual neto (VAN), tasa interna de retorno (TIR), índice de valor actual neto (IVAN), razón beneficio-costo (RBC) y período de recuperación del capital (PRC). Se utilizaron las expresiones (Campos et al., 1995; Fontaine, 1997; Lobos et al., 2001):

$$
\begin{aligned}
& \text { VAN }=-I_{0}+\sum_{i=0}^{n} \frac{F^{2 N C_{i}}}{(1+r)^{i}}(1) \\
& 0=-I_{0}+\sum_{i=0}^{n} \frac{\text { FNC }_{i}}{(1+\Phi)^{i}}(2)
\end{aligned}
$$

$$
\begin{aligned}
& \text { IVAN }=\frac{\text { VAN }}{I_{0}}(3) \\
& R B C=\frac{\text { VAY }}{\text { VAC }}(4),
\end{aligned}
$$

donde $I_{0}$ representa la inversión inicial, $\mathrm{n}$ es el horizonte de evaluación del proyecto, FNC es el flujo neto de caja, $r$ es la tasa de descuento relevante, $\Phi$ es la TIR, VAY es el valor actual de los ingresos y VAC es el valor actual de los costos (egresos). El PRC se obtuvo como el año en que el FNC actualizado y acumulado es mayor que cero. En los costos totales se incluyeron los costos directos, gastos generales y administración y ventas, contribuciones, impuesto a la renta de $1^{\text {a }}$ categoría y el costo alternativo del arriendo del terreno agrícola.

Para medir la eficiencia económica se calculó el costo medio total (CMET), margen unitario ponderado (MUP) y la rentabilidad sobre el capital de explotación (RSC), los cuales fueron proyectados durante el horizonte de evaluación definido para el proyecto. Se usaron las expresiones (Campos et al., 1995; Fontaine, 1997; Lobos et al., 2001):

$$
\begin{gathered}
\text { CMET }=\frac{\text { CTP }}{Q}(5) \\
M U P=P P P-C M E T \\
\text { RSC }=\frac{U T}{C E X}(7),
\end{gathered}
$$

donde CT es el costo total de producción, Q es el volumen de producción, PPP es el precio de venta promedio ponderado (considerando como factor de ponderación el porcentaje de ventas en el mercado interno y externo), UT es la utilidad y CEX es el capital de explotación. Este último se calculó como el valor actual de la plantación (establecimiento y formación), y el valor de la tierra, considerando su tasación comercial (Vargas, 2004).

\section{Coeficientes técnicos}

Considerando que cada temporada agrícola se inicia en mayo y termina en abril del año siguiente, para efectos del horizonte de planeación del proyecto, los datos técnicos fueron estructurados 
de la siguiente forma: el año 0 (establecimiento del cultivo) incluyó desde mayo a diciembre de la primera temporada agrícola; el año 1 (formación del cultivo) desde enero a abril de la primera temporada agrícola; como año 2 (inicio de la etapa de producción del cultivo) se consideró la segunda temporada agrícola (desde mayo a abril del año siguiente), y así sucesivamente. Desde el punto de vista económico, el período que comprende los años 0 y 1 del proyecto (sin producción) corresponde a la fase de inversión y desde el año 2 en adelante corresponde a la etapa de producción. Los coeficientes técnicos utilizados para el horizonte de planeación del proyecto se presentan en las Tablas 1 y 2.

\section{Valores unitarios}

La valorización de las inversiones y costos se realizó a partir de los valores promedio por unidad pagados por el predio durante el período de análisis, todos los cuales fueron convertidos a moneda de septiembre de 2004 (Tablas 1 y 2).

- Mano de obra directa permanente: el valor de la JH fue estimado a partir de la remuneración bruta mensual pagada a la mano de obra de planta, la cual corresponde a la retribución por el trabajo realizado en todo el predio. Por lo tanto, a partir del número de ha atendidas, cada tipo de mano de obra permanente fue llevado a $\mathrm{JH} \mathrm{ha}^{-1}$.

- Mano de obra directa temporal: se consideraron dos componentes, en función de la disponibilidad de mano de obra: (a) una remuneración correspondiente al salario mínimo, más otros gastos pagados durante seis meses de cada temporada agrícola y; (b) una remuneración pagada durante los restantes seis meses de la misma temporada agrícola, en la cual se incluyó una remuneración fija y una asignación de movilización.

- Insumos agrícolas: los precios de los insumos agrícolas corresponden a los valores pagados por el predio, sin impuestos. En el caso de las plantas nuevas, el valor de cada planta de espárrago se estimó en Ch \$18,50 (sin impuestos).

- Servicios de maquinaria y equipos: el costo unitario, medido en JM $\mathrm{ha}^{-1}$, fue estimado a partir de los precios promedio de mercado, sin impuestos, pagados por el predio por concepto de arriendo de maquinaria y equipos. El valor de uso de los servicios de maquinaria y equipos propios fue estimado a partir del prorrateo de los costos totales incurridos en el predio.

- Gastos generales y gastos administrativos: fueron estimados en base a los valores pagados por el predio, prorrateados por 1 ha.

\section{Valorización de las inversiones y} costos

La valorización de las inversiones en plantación fue realizada de acuerdo a lo establecido por el Servicio de Impuestos Internos (SII) en la Circular $\mathrm{n}^{\text {o. }} 28$, del 28 de mayo de 1982, bajo cuyo sistema las explotaciones agrícolas quedan sujetas al impuesto a la renta de $1^{\text {a. }}$ categoría del $17 \%$. En las inversiones se incluyó mano-de-obra directa permanente y temporal; insumos agrícolas; servicios de maquinaria y equipos; gastos generales; gastos de administración y ventas, e imprevistos. La inversión en plantación, de acuerdo a la citada Circular, se depreció a su valor libro en 6 años (año 5 al 10). Considerando que desde el punto de vista del inversionista interesa conocer la viabilidad económica de la plantación, se incluyó el valor de uso del terreno agrícola, después de impuestos, como el costo alternativo del arriendo. La clasificación de costos de producción se realizó de acuerdo a la propuesta por González (1998), Velasco et al. (2000) y Vargas (2004).

\section{Ingresos por ventas}

Los datos de productividad fueron obtenidos de los registros históricos del predio, de acuerdo a los niveles de producción promedio alcanzados durante cada temporada agrícola. Los ingresos por ventas fueron estimados a partir del destino de la producción, suponiendo un $30 \%$ al mercado interno y un $70 \%$ al mercado externo. Los precios de venta en cada mercado fueron estimados de acuerdo al siguiente procedimiento:

- Mercado interno: se tomó el precio real promedio del período 2000 a 2003 en el mercado mayorista, reportado por ODEPA (2004). Se consideró un precio promedio de venta de Ch $\$ 549,50 \mathrm{~kg}^{-1}$ (equivalente a US \$ $0,92 \mathrm{~kg}^{-1}$ ), sin impuestos, para las ventas de espárragos frescos en el mercado interno.

- Mercado externo: se consideró un precio real promedio a productor de US\$1,33 $\mathrm{kg}^{-1}$, equivalente a Ch\$ 799,90 $\mathrm{kg}^{-1}$ (US\$ $\left.1.00=\mathrm{Ch} \$ 600=\mathrm{US} \$ 1\right)$. Este precio fue obtenido a partir de los precios medios FOB, una vez descontada la comisión de exportación y las tarifas cobradas por la empresa exportadora (servicios de embalaje, frío, control de calidad y flete desde la planta al puerto de embarque, entre otros). El precio neto a productor fue convertido a moneda (qual moeda) de septiembre de 2004, utilizando como deflactor el Índice de Precios de Importación (Base septiembre $2000=100$ ), reportado por la Oficina de Estadísticas Laborales (BLS, 2004) de EE.UU.

\section{Análisis de sensibilidad}

Los valores anteriores permitieron conocer la viabilidad económica del proyecto en la situación estándar. Para las ventas en el mercado externo, se sensibilizó el precio neto recidido por el productor de $\mathrm{Ch} \$ 799,9 \mathrm{~kg}^{-1} \pm$ el porcentaje de variabilidad de la serie de precios reales FOB entre enero 2000 y septiembre de 2004, el cual fue estimado en $15,8 \%$. Además, se sensibilizó la productividad del proyecto considerando una variación de $\pm 5 \%$, de acuerdo a los rendimientos históricos obervados.

\section{Tasa de descuento}

Como tasa de descuento se utilizó el costo de capital promedio ponderado (WACC $=8,71 \%$ ) reportado por Lobos \& Muñoz (2005) para la evaluación económica de proyectos de plantaciones permanentes. Para esta investigación, dicha tasa representa el retorno económico mínimo exigible al cultivo de espárrago.

\section{RESULTADOS Y DISCUSIÓN}

El costo total de establecimiento del huerto de espárrago (año 0) se estimó en Ch\$1,89 millones ha-1 (Tabla 3). El $39 \%$ corresponde a la adquisición de plantas y el $18 \%$ a la contratación de 
Tabla 1. Costos de producción de 1 ha de espárragos en la Región del Maule, Chile, año 0-5, en Pesos Chilenos ${ }^{1}$. Linares, Universidad de Talca, 2004

\begin{tabular}{|c|c|c|c|c|c|c|c|c|c|c|c|c|c|}
\hline \multirow[b]{2}{*}{ Componentes } & \multirow[b]{2}{*}{ Unidad } & \multicolumn{2}{|c|}{ año 0} & \multicolumn{2}{|c|}{ año 1} & \multicolumn{2}{|c|}{ año 2} & \multicolumn{2}{|c|}{ año 3} & \multicolumn{2}{|c|}{ año 4} & \multicolumn{2}{|c|}{ año 5} \\
\hline & & $\begin{array}{l}\text { Can- } \\
\text { tidad }\end{array}$ & Costo (\$) & $\begin{array}{c}\text { Canti- } \\
\text { dad }\end{array}$ & Costo (\$) & $\begin{array}{l}\text { Canti- } \\
\text { dad }\end{array}$ & Costo (\$) & $\begin{array}{c}\text { Canti- } \\
\text { dad }\end{array}$ & Costo $(\$)$ & $\begin{array}{c}\text { Canti- } \\
\text { dad }\end{array}$ & Costo $(\$)$ & $\begin{array}{c}\text { Canti- } \\
\text { dad }\end{array}$ & Costo (\$) \\
\hline Permanentes & $\mathrm{JH}$ & 48 & 340.000 & 24 & 170.000 & 72 & 510.000 & 72 & 510.000 & 72 & 510.000 & 72 & 510.000 \\
\hline Temporales & $\mathrm{JH}$ & 54 & 338.495 & 93 & 588.411 & 42 & 267.379 & 73 & 459.720 & 91 & 574.049 & 98 & 617.389 \\
\hline Máquina propia & $\mathrm{h}$ & 20 & 84.000 & 4 & 14.700 & 12 & 29.400 & 12 & 29.400 & 12 & 29.400 & 12 & 29.400 \\
\hline $\begin{array}{l}\text { Máquina } \\
\text { arriendo }\end{array}$ & $\mathrm{JM}$ & 0 & 0 & 0 & 0 & 5 & 330.000 & 5 & 330.000 & 5 & 330.000 & 5 & 330.000 \\
\hline $\begin{array}{l}\text { Subtotal } \\
\text { servicios }\end{array}$ & & & 762.495 & & 773.111 & & 1.136 .779 & & 1.329 .120 & & 1.443 .449 & & 1.486 .789 \\
\hline Fertilizantes & $\mathrm{kg}$ & 350 & 54.250 & 1.004 & 160.716 & 1.304 & 160.716 & 1.304 & 160.716 & 1.304 & 160.716 & 1.304 & 160.716 \\
\hline Insecticidas & $\mathrm{L}$ & 0 & 0 & 5 & 61.945 & 5 & 38.087 & 7 & 74.474 & 7 & 74.474 & 8 & 76.434 \\
\hline Herbicidas & L & 0 & 0 & 0 & 0 & 32 & 116.682 & 31 & 107.106 & 31 & 107.106 & 31 & 107.106 \\
\hline $\begin{array}{l}\text { Subtotal } \\
\text { insumos }\end{array}$ & & & 54.250 & & 222.661 & & 315.485 & & 342.296 & & 342.296 & & 344.256 \\
\hline Generales $^{2}$ & $\mathrm{~N}^{\circ}$ & 20 & 158.304 & 10 & 113.522 & 33 & 327.147 & 33 & 338.104 & 33 & 343.821 & 33 & 346.086 \\
\hline Administrativos & $\mathrm{N}^{\circ}$ & 8 & 140.000 & 4 & 70.000 & 12 & 210.000 & 12 & 210.000 & 12 & 210.000 & 12 & 210.000 \\
\hline Subtotal otros & & & 298.304 & & 183.522 & & 537.147 & & 548.104 & & 553.821 & & 556.086 \\
\hline Costos totales $^{3}$ & & & 1.892 .048 & & 1.179 .294 & & 1.989 .410 & & 2.219 .520 & & 2.339 .566 & & 2.387 .130 \\
\hline Productividad & (kg/ha) & 0 & & 0 & & 1.000 & & 3.500 & & 4.500 & & 5.000 & \\
\hline
\end{tabular}

${ }^{1} \mathrm{US} \$ 1,00=600$ pesos chilenos; ${ }^{2}$ Incluye $\mathrm{Ch} \$ 30.000$ por concepto de pago de contribuciones, equivalente al $2 \%$ del avalúo fiscal del terreno agrícola, e imprevistos, equivalente al $5 \%$ de la suma de los costos de servicios e insumos; ${ }^{3}$ En el año 0 incluye Ch\$740.000 por concepto de inversión en plantas, calculado como 40.000 plantas a un valor de Ch\$18,5 cada una.

Tabla 2. Costos de producción de 1 ha de espárragos en la Región del Maule, Chile, año 6-10, en Pesos Chilenos ${ }^{1}$. Linares, Universidad de Talca, 2004.

\begin{tabular}{|c|c|c|c|c|c|c|c|c|c|c|c|}
\hline \multirow{2}{*}{ Componentes } & \multirow{2}{*}{ Unidad } & \multicolumn{2}{|c|}{ año 6} & \multicolumn{2}{|c|}{ año 7} & \multicolumn{2}{|c|}{ año 8} & \multicolumn{2}{|c|}{ año 9} & \multicolumn{2}{|c|}{ año 10} \\
\hline & & Cantidad & Costo (\$) & Cantidad & Costo (\$) & Cantidad & Costo (\$) & Cantidad & Cos to $(\$)$ & Cantidad & Costo (\$) \\
\hline Permanentes & $\mathrm{JH}$ & 72 & 510.000 & 72 & 510.000 & 72 & 510.000 & 72 & 510.000 & 72 & 510.000 \\
\hline Temporales & $\mathrm{JH}$ & 103 & 649.150 & 111 & 702.930 & 121 & 763.036 & 130 & 821.245 & 139 & 880.086 \\
\hline Máquina propia & $\mathrm{h}$ & 12 & 29.400 & 12 & 29.400 & 12 & 29.400 & 12 & 29.400 & 12 & 29.400 \\
\hline $\begin{array}{l}\text { Máquina } \\
\text { arriendo }\end{array}$ & $\mathrm{JM}$ & 5 & 330.000 & 5 & 330.000 & 5 & 330.000 & 5 & 330.000 & 5 & 330.000 \\
\hline $\begin{array}{l}\text { Subtotal } \\
\text { servicios }\end{array}$ & & & 1.518 .550 & & 1.572 .330 & & 1.632 .436 & & 1.690 .645 & & 1.749 .486 \\
\hline Fertilizantes & $\mathrm{kg}$ & 1.304 & 160.716 & 1.304 & 160.716 & 1.304 & 160.716 & 1.304 & 160.716 & 1.304 & 160.716 \\
\hline Insecticidas & $\mathrm{L}$ & 7 & 74.474 & 7 & 74.474 & 7 & 74.474 & 7 & 74.474 & 7 & 74.474 \\
\hline Herbicidas & $\mathrm{L}$ & 31 & 107.106 & 31 & 107.106 & 31 & 107.106 & 31 & 107.106 & 31 & 107.106 \\
\hline $\begin{array}{l}\text { Subtotal } \\
\text { insumos }\end{array}$ & & & 342.296 & & 342.296 & & 342.296 & & 342.296 & & 342.296 \\
\hline Generales2 & $\mathrm{N}^{\circ}$ & 33 & 347.576 & 33 & 350.265 & 33 & 353.270 & 33 & 356.180 & 33 & 359.122 \\
\hline Administrativos & $\mathrm{N}^{\circ}$ & 12 & 210.000 & 12 & 210.000 & 12 & 210.000 & 12 & 210.000 & 12 & 210.000 \\
\hline Subtotal otros & & & 557.576 & & 560.265 & & 563.270 & & 566.180 & & 569.122 \\
\hline Costos totales & & & 2.418 .422 & & 2.474 .891 & & 2.538 .002 & & 2.599.121 & & 2.660 .904 \\
\hline Productividad & $(\mathrm{kg} / \mathrm{ha})$ & 6.000 & & 7.000 & & 8.000 & & 9.000 & & 9.000 & \\
\hline
\end{tabular}

${ }^{1}$ US $\$ 1,00=600$ pesos chilenos; ${ }^{2}$ Incluye Ch $\$ 30.000$ por concepto de pago de contribuciones, equivalente al $2 \%$ del avalúo fiscal del terreno agrícola, e imprevistos, equivalente al $5 \%$ de la suma de los costos de servicios e insumos.

mano-de-obra temporal. El costo total durante la etapa de formación (año 1) alcanza a Ch\$ 1,18 millones ha ${ }^{-1}$. La mano-de-obra temporal representa el
$50 \%$ de dicho costo. En la etapa de producción, la incidencia de las labores de cultivo temporal dentro de los costos directos aumenta desde un 18\% (año 3) hasta un $42 \%$ (año 10), mientras que la participación de los insumos agrícolas disminuye desde un $22 \%$ (año 3 ) hasta un $16 \%$ (año 10). Esta estructura de 
Tabla 3. Flujo neto de caja de la producción de 1 ha de espárragos en la Región del Maule, Chile, año 0-10, en Pesos Chilenos ${ }^{1}$. Linares, Universidad de Talca, 2004.

\begin{tabular}{|c|c|c|c|c|c|c|c|c|c|c|c|}
\hline Componentes & año 0 & año 1 & año 2 & año 3 & año 4 & año 5 & año 6 & año 7 & año 8 & año 9 & a ño 10 \\
\hline $\begin{array}{l}\text { Inversión } \\
\text { plantación }\end{array}$ & -1.892 .048 & -1.179 .294 & & & & & & & & & \\
\hline $\begin{array}{l}\text { Inversión capital } \\
\text { de trabajo }\end{array}$ & & -1.452 .264 & & & & & & & & & 1.452 .264 \\
\hline $\begin{array}{l}\text { Ingresos mercado } \\
\text { nacional }\end{array}$ & & & 164.854 & 576.988 & 741.842 & 824.269 & 989.123 & 1.153 .976 & 1.318 .830 & 1.483 .684 & 1.483 .684 \\
\hline $\begin{array}{l}\text { Ingresos } \\
\text { me rcad o extemo }\end{array}$ & & & 559.934 & 1.959 .770 & 2.519 .704 & 2.799 .671 & 3.359 .606 & 3.919 .540 & 4.479 .474 & 5.039 .408 & 5.039 .408 \\
\hline $\begin{array}{l}\text { Costos servicios } \\
\text { e insumos }\end{array}$ & & & -1.452 .264 & -1.671 .416 & -1.785 .745 & -1.831 .045 & -1.860 .846 & -1.914 .626 & -1.974 .732 & -2.032 .941 & -2.091 .782 \\
\hline $\begin{array}{l}\text { Margem } \\
\text { bruto }\end{array}$ & & & -727.476 & 865.342 & 1.475 .801 & 1.792 .895 & 2.487 .882 & 3.158 .890 & 3.823 .572 & 4.490 .151 & 4.431 .310 \\
\hline $\begin{array}{l}\text { Gastos generales } \\
\text { y adm. }\end{array}$ & & & -537.147 & -548.104 & -553.821 & -556.086 & -557.576 & -560.265 & -563.270 & -566.180 & -569.122 \\
\hline $\begin{array}{l}\text { Depreciación } \\
\text { plantación }\end{array}$ & & & & & & -511.890 & -511.890 & -511.890 & -511.890 & -511.890 & -511.890 \\
\hline $\begin{array}{l}\text { Utilidad } \\
\text { (Pérdida) }\end{array}$ & & & -1.264 .622 & 317.238 & 410.090 & 724.919 & 1.418 .416 & 2.086 .735 & 2.748 .411 & 3.412 .080 & 3.862 .188 \\
\hline $\begin{array}{l}\text { Pérdida } \\
\text { ejercicio anterior }\end{array}$ & & & & -1.264 .622 & -947.384 & -537.294 & & & & & \\
\hline $\begin{array}{l}\text { Utilidad } \\
\text { afecta }\end{array}$ & & & -1.264 .622 & -947.384 & -537.294 & 187.625 & 1.418 .416 & 2.086 .735 & 2.748 .411 & 3.412 .080 & 3.862 .188 \\
\hline Impue sto $(17 \%)$ & & & 0 & 0 & 0 & -31.896 & -241.131 & -354.745 & -467.230 & -580.054 & -656.572 \\
\hline Utilidad neta & & & -1.264 .622 & 317.238 & 410.090 & 693.023 & 1.177285 & 1.731 .990 & 2.281 .181 & 2.832 .027 & 3.205 .616 \\
\hline $\begin{array}{l}\text { Depreciación } \\
\text { plantación }\end{array}$ & & & & & & 511.890 & 511.890 & 511.890 & 511.890 & 511.890 & 511.890 \\
\hline $\begin{array}{l}\text { Costo altemativo } \\
\text { del terreno }\end{array}$ & -261.300 & -261.300 & -261.300 & -261.300 & -261.300 & -261.300 & -261.300 & -261.300 & -261.300 & -261.300 & -261.300 \\
\hline $\begin{array}{l}\text { Flujo neto de } \\
\text { caja }\end{array}$ & -2.153 .348 & -2.892 .858 & -1.525 .922 & 55.938 & 148.790 & 943.613 & 1.427 .875 & 1.982 .581 & 2.531 .772 & 3.082 .617 & 4.908 .470 \\
\hline
\end{tabular}

${ }^{1} \mathrm{US} \$ 1,00=600$ pesos chilenos

costos es consistente con la reportada por Vargas (2004) para el caso de los cultivos permanentes y por Lobos \& Muñoz (2005) para las plantaciones de manzano.

El valor actual de la plantación de espárrago se estimó en $\mathrm{Ch} \$ 2,98$ millones $\mathrm{ha}^{-1}$, para la densidad de plantación de 40.000 plantas ha ${ }^{-1}$ considerada en este trabajo, la cual está dentro de los rangos reportados por diferentes estudios (Kaufmann \& Orth, 1990; Loughton et al., 1996; Bussell et al., 1997; González \& Del Pozo, 1999). Para productores con tierra, en todos los escenarios considerados los indicadores de viabilidad sugieren que el cultivo de espárrago es un proyecto rentable, excepto cuando se tomó el precio en el mercado externo reducido en el porcentaje de variabilidad de los precios internacionales (Tabla 4). Por lo tanto, bajo condiciones normales de precios de venta y productividad (ceteris paribus las otras variables), en general los resultados indican que, desde el punto de vista económico, el cultivo de espárrago permitiría generar un "incremento en riqueza" (medido en valor actual o valor anual equivalente) para los inversionistas privados dado que el VAN es mayor que cero; la TIR es mayor que la tasa de descuento utilizada, por lo que el proyecto podría "soportar" todavía una mayor exigencia en términos del costo alternativo del capital ("second best"); el IVAN mayor que cero muestra que por cada unidad monetaria invertida en este cultivo se obtiene "riqueza"; la RBC refleja que por cada unidad monetaria de costo (egreso) es posible alcanzar más que esa unidad monetaria de ingreso; el PRC fluctúa entre 9 y 10 años, por lo que el proyecto, aunque lentamente, permitiría recuperar la inversión dentro del horizonte de evaluación considerado. Los indicadores de eficiencia económica proyectados muestran que el cultivo de espárrago permitiría lograr una reducción gradual de los costos unitarios de producción hasta alcanzar valores entre Ch\$302,70 y 334,60 kg-1; además se lograrían márgenes unitarios crecientes de entre $\mathrm{Ch} \$ 390,20$ y 422,10 $\mathrm{kg}^{-1}$; la rentabilidad sobre el capital de explotación que podría alcanzar un productor fluctuaría entre 18,1 y 25,5\%.

Los resultados obtenidos en esta investigación sugieren que en la misma zona de estudio la inversión en el cultivo de espárrago es menos rentable que la plantación de frutales (Lobos \& Muñoz, 2005), aunque es más rentable que la inversión en lecherías (Campos et al., 1995; Lobos et al., 2001). Por ejemplo, en el trabajo de Lobos \& Muñoz (2005) la RSC se proyectó, en plena producción, en un rango entre 11,4 y $35,9 \%$, incluyendo el análisis de distintos escenarios de precios de venta, tipo de cambio, rendimiento y tasa de descuento. 
Tabla 4. Indicadores de viabilidad y eficiencia económica de la producción de 1 ha de espárrago en la Región del Maule, Chile, en Pesos Chilenos ${ }^{1}$. Linares, Universidad de Talca, 2004.

\begin{tabular}{|c|c|c|c|c|c|}
\hline Componentes & $\begin{array}{l}\text { Situación } \\
\text { estándar }\end{array}$ & $\begin{array}{c}\text { Precio alto en } \\
\text { mercado externo }\end{array}$ & $\begin{array}{c}\text { Precio bajo en } \\
\text { mercado externo }\end{array}$ & $\begin{array}{l}\text { Productividad } \\
\text { más alta }\end{array}$ & $\begin{array}{c}\text { Productividad } \\
\text { más baja }\end{array}$ \\
\hline Precio mercado externo ( $\$ / \mathrm{kg})$ & 799,9 & 926,4 & 673,4 & 799,9 & 799,9 \\
\hline Productividad (kg/ha) & Nomal & Nomal & Nomal & $5 \%$ más alta & $5 \%$ más baja \\
\hline Valor actual neto $(\$)$ & 1.517 .144 & 3.726 .106 & -691.818 & 2.420 .999 & 613.289 \\
\hline Valor anual equivalente $(\$)$ & 233.393 & 573.212 & -106.427 & 372.439 & 94.346 \\
\hline Tasa interna de retomo (\%) & 12,1 & 16,7 & 7,0 & 14,1 & 10,1 \\
\hline Índice de valor actual neto $(\$)$ & 0,3 & 0,6 & $-0,1$ & 0,41 & 0,10 \\
\hline Razón beneficio a costo $(\$)$ & 1,1 & 1,2 & 0,9 & 1,14 & 1,03 \\
\hline Período recuperación del capital (años) & 10 & 9 & no recupera & 9 & 10 \\
\hline Precio venta promedio ponderado $(\$ / \mathrm{kg})$ & 724,8 & 813,4 & 636,2 & 724,8 & 724,8 \\
\hline \multicolumn{6}{|l|}{ Costo medio total $(\$ / \mathrm{kg})$ : } \\
\hline Año 3 & 708,8 & 708,8 & 708,8 & 675,1 & 746,1 \\
\hline Año 4 & 578,0 & 578,0 & 578,0 & 550,4 & 608,4 \\
\hline Año 5 & 529,7 & 529,7 & 529,7 & 504,5 & 557,6 \\
\hline Año 6 & 446,6 & 446,6 & 446,6 & 425,4 & 470,1 \\
\hline Año 7 & 390,9 & 390,9 & 390,9 & 372,3 & 411,5 \\
\hline Año 8 & 349,9 & 349,9 & 349,9 & 333,3 & 368,3 \\
\hline Año 9 & 317,8 & 317,8 & 317,8 & 302,7 & 334,6 \\
\hline \multicolumn{6}{|l|}{ Margen unitario ponderado $(\$ / \mathbf{k g})$ : } \\
\hline Año 3 & 16,0 & 104,5 & $-72,6$ & 49,7 & $-21,3$ \\
\hline Año 4 & 146,8 & 235,4 & 58,3 & 174,3 & 116,4 \\
\hline Año 5 & 195,1 & 283,7 & 106,5 & 220,3 & 167,2 \\
\hline Año 6 & 278,2 & 366,7 & 189,6 & 299,4 & 254,7 \\
\hline Año 7 & 333,9 & 422,5 & 245,3 & 352,5 & 313,3 \\
\hline Año 8 & 374,9 & 463,4 & 286,3 & 391,5 & 356,5 \\
\hline Año 9 & 407,0 & 495,5 & 318,4 & 422,1 & 390,2 \\
\hline \multicolumn{6}{|l|}{ Rentabilidad sobre c apital (\%): } \\
\hline Año 3 & 0,0 & 3,4 & $-3,3$ & 1,4 & $-1,3$ \\
\hline Año 4 & 6,0 & 10,0 & 2,1 & 7,7 & 4,4 \\
\hline Año 5 & 8,4 & 12,5 & 4,4 & 10,1 & 6,8 \\
\hline Año 6 & 9,3 & 13,8 & 4,8 & 11,1 & 7,5 \\
\hline Año 7 & 13,7 & 18,5 & 8,9 & 15,7 & 11,8 \\
\hline Año 8 & 17,3 & 22,4 & 12,3 & 19,4 & 15,3 \\
\hline Año 9 & 20,3 & 25,5 & 15,1 & 22,4 & 18,1 \\
\hline
\end{tabular}

${ }^{1} \mathrm{US} \$ 1,00=600$ pesos chilenos.

Los resultados de esta investigación sugieren que el cultivo de espárrago verde en Chile es un negocio económicamente viable y, por lo tanto, rentable. Sin embargo, los productores nacionales deberían centrar sus esfuerzos de comercialización hacia los mercados internacionales, donde es posible obtener mayores precios para el producto, y por lo tanto, una mayor rentabilidad del cultivo.

\section{AGRADECIMIENTOS}

Los autores agradecen a la Facultad de Ciencias Empresariales de la Universidad de Talca por el apoyo financiero para la publicación de este trabajo, a través del Fondo de Desarrollo Académico.

\section{REFERÊNCIAS}

BLS - Bureau of Labor Statistics. 2000, 19 de agosto. Import price indexes for selected categories of goods. Disponible en http:// www.bls.gov/mxp/home.htm

BUSSELL WT; MAINDONALD JH; MORTON JR. 1997. What is a correct plant density for transplanted green asparagus? New Zealand Journal of Crop and Horticultural Science 25: 359-368.

CAMPOS MA; BECK A; HAUSDORF R. 1995. Análisis económico de la producción lechera en predios de la zona central de Chile. Agricultura Técnica 55: 140-146.
DEL POZOA. 1999. Morfología y funcionamiento de la planta. In: GONZÁLEZ MI; DEL POZO A. (eds). El cultivo del espárrago. Chillán: INIA. p. 9-28 (Boletín n ${ }^{\circ}$ 6).

FAOSTAT - FAO Statistical Database. Agricultural production. 2005, 15 de noviembre. Available at http://faostat.fao.org/faostat/ collections?version=ext\&hasbulk=0\&subset=agriculture

FERRARI V; PORCELLI S; FICCADENTI N; ACCIARRI N. 1990. Results of plant density on quantity and quality early yields in green asparagus (Asparagus officinalis L.) Acta Horticulturae 271: 243-248.

FONTAINE E. 1997. Evaluación social de proyectos. $11^{\mathrm{a}}$ ed. Santiago: Ediciones Pontificia Universidad Católica de Chile. 466p.

FRANCE A. 1999. Enfermedades. In: GONZÁLEZ MI; DEL POZO A. (eds). El cultivo del espárrago. Chillán: INIA. p. 125164 (Boletín no. 6). 
GONZÁLEZ MI. 1998. Fichas hortícolas para el área Centro-Sur VII y VIII regiones. Chillán: INIA. 54p. (Boletín INIA Quilamapu n ${ }^{\circ}$ 104).

GONZÁLEZ MI. 1999. Variedades. In: GONZÁLEZ MI; DEL POZO A. (eds). El cultivo del espárrago. Chillán: INIA. p. 53-63 (Boletín $n^{\circ}$ 6).

GONZÁlEZ MI; DEL POZO, A. 1999. Establecimiento de la esparraguera. In: GONZÁLEZ MI; DEL POZO A. (eds). El cultivo del espárrago. Chillán: INIA. p. 65-78 (Boletín no. 6).

GONZÁLEZ MI; DEL POZO A. 2003. Efectos de la profundidad de plantación y población de plantas en la calidad y rendimiento de espárrago verde. Agricultura Técnica 63: 223-230.

GTI - Global Trade Information. 2004, 15 de noviembre. Trade statistics. Disponible en http://www.gtis.com/

KAUFMANNF; ORTHWD. 1990. Principles of plant density for green asparagus harvested by different methods. Acta Horticulturae 271: 227-234.

KRARUP C; CONTRERAS S. 2001. Elongación y ramificación de turiones de espárrago durante una cosecha primaveral. Agricultura Técnica 62:191-200.
LOBOS G; SOTO R; ZENTENO N; PRIZANT A. 2001. Análisis de eficiencia y rentabilidad económica en dos lecherías de la Región del Maule, Chile. Agricultura Técnica 61: 367-378.

LOBOS G; MUÑOZ T. 2005. Indicadores de rentabilidad y eficiencia económica de la producción de manzanas (Malus domestica L.) Gala en la Región del Maule, Chile. Agricultura Técnica 65:421-436.

LOUGHTON A; BAKER R; ALLEN OB. 1996. Yield and growth responses of asparagus to between-row spacing and planting depth. Canadian Journal of Plant Science 76: 841-847.

ODEPA - Oficina de Estudios y Políticas Agrarias. 2004, 12 de noviembre. Base de datos de comercio exterior. Disponible en http:// www.odepa.cl

ORTEGA R. 1999. Manejo de la fertilización. In: GONZÁLEZ MI; DEL POZO A. (eds). El cultivo del espárrago. Chillán: INIA. p. 79-96 (Boletín $\mathrm{n}^{\mathrm{o}}$ 6)

PIGNON P, CORBAZ RO; PERKO J. 1994. Asperge verte: resultats d'experimentation durant sept ans de culture. Revue Suisse de Viticulture, d'Arboriculture et d'Horticulture 2691-96.
USDA - United States Department of Agriculture. 2004, 15 noviembre. World asparagus situation \& outlook. Disponible en http:// Www.fas.usda.gov/

VARGAS G. 2004, 15 noviembre. Manual de criterios comunes para el control de gestión en empresas agropecuarias. Santiago: Fundación Chile y Pontificia Universidad Católica de Chile. Disponible en http:// ww w.agrogestion.cl/docs-agro/ ManualCriteriosComunes.pdf

VELASCO R; AVILÉS R. 1999. Costos de producción y análisis de sensibilidad y rentabilidad. In: GONZÁLEZ MI; DEL POZO A. (eds). El cultivo del espárrago. Chillán, Chile: Boletín INIA Nº, p.181-200.

VELASCO R; GONZÁLEZ M; GONZÁLEZ J; TAY J. 2000. Estándares técnicos y resultados económicos de hortalizas en la VII y VIII regiones. Chillán: INIA. 69p. (Boletín $\mathrm{n}^{\circ}$ 17). 